\title{
OBJETO DE APRENDIZAGEM: TEORIA INSTRUTIVA APOIADA POR COMPUTADOR
}

\author{
Leila Maria Araújo Santos - Ulbra/SM, PGIE/CINTED/UFRGS \\ leilamas@terra.com.br \\ Maria Lucia Pozzatti Flores - Ulbra/SM, PGIE/CINTED/UFRGS \\ mlflores@terra.com.br
}

\author{
Liane Margarida Rockenbach Tarouco - CINTED/UFRGS \\ liane@penta.ufrgs.br
}

\begin{abstract}
Resumo: Este artigo relata um estudo que está sendo realizado sobre a teoria instrutivista LODAS, para que seus princípios sejam implementados futuramente no desenvolvimento de um Objeto de Aprendizagem para ensinar a Fórmula de Bhaskara. O Objeto de Aprendizagem proposto visa aproximar a tecnologia ao ensino, favorecendo, ao estudante, a aquisição de conhecimentos, competências e habilidades na resolução da equação do segundo grau, usando a Fórmula de Bhaskara, tornando a aprendizagem mais dinâmica e atraente.
\end{abstract}

Palavras-chave: Teoria instrutivista, Objeto de Aprendizagem

Abstract: This article tells a study that is being accomplished on the theory instructive LODAS, so that their beginnings are implemented hereafter in the development of an Object of Learning to teach the Formula of Bhaskara. The Object of Learning proposed seeks to approximate the technology to the teaching, favoring, to the student, the acquisition of knowledge, competences and abilities in the resolution of the equation of the second degree, using the Formula of Bhaskara, turning the most dynamic and attractive learning.

Keywords: Theory instructive, Object of Learning

\section{Introdução}

A tecnologia é um agente de mudança e a maioria das inovações tecnológicas podem resultar em uma revolucionária quebra de paradigma educacional. Sabe-se que a rede mundial de computadores, conhecida como Internet, é uma dessas inovações. Após influenciar a forma como as pessoas se comunicam e fazem negócios, a Internet também vem modificando o processo de ensino e aprendizagem, e trazendo novas descobertas sobre a forma como as pessoas aprendem. Consequientemente, estes novos conhecimentos geram mudanças de forma e conteúdo da educação, e uma maior mudança vem sendo notada no desenvolvimento de recursos educacionais desenvolvidos e integrados para serem utilizados e disponibilizados no ensino.

Existem diversos enfoques apresentados por pesquisadores em tecnologia educacional, sendo que a maioria compartilha um enfoque comum de que a composição das partes dos cursos virtuais, total ou parcial, deve ser pequena, digital e com capacidade para a reutilização, para que efetivamente possa ser um recurso educacional. Estes recursos educacionais podem ser chamados de objetos de aprendizagem, de 
acordo com a terminologia adotada pelo Learning Technology Standards Committee (LTSC) do Institute of Electrical and Electonics Engineers (IEEE).

\section{Objeto de Aprendizagem}

O objeto de aprendizagem surgiu com o objetivo de localizar conteúdos educacionais na Web, para serem reutilizados em diferentes cursos e plataformas, possibilitando, assim, a redução de custos de produção de materiais educacionais.

Segundo IEEE(2000), o objeto de aprendizagem é definido como qualquer entidade, que pode ser utilizada, reutilizada ou referenciada durante o aprendizado apoiado por computador. Ele pode conter simples elementos como um texto ou um vídeo. Ou ainda, ele pode ser um hipertexto, um curso ou até mesmo uma animação com áudio e recursos mais complexos.

Wiley (2000) utiliza a metáfora de um átomo para explicar o objeto de aprendizagem. Ele explica que um átomo é um elemento pequeno que pode ser combinado e recombinado com outros elementos pequenos formando algo maior. $\mathrm{Ou}$ seja, cada objeto de aprendizagem pode constituir-se em um módulo com um conteúdo auto-explicativo, de sentido complemento. Este também pode ser direcionado a outros módulos para formar um curso mais abrangente. $\mathrm{O}$ autor acrescenta, também, que um átomo não pode ser recombinado com qualquer outro tipo de átomo. Estes têm que estar dentro do mesmo contexto, isto é, conter conteúdos que se relacionam entre si. Wiley (2000) entende que os objetos de aprendizagem devem ter as seguintes características: serem auto-explicativos, modulares, agregáveis, digitais, interoperáveis e reutilizáveis.

Para facilitar a adoção dos objetos de aprendizagem, o Learning Technology Standards Committee (LTSC) do IEEE, em 1996, forneceu padrões instrutivos da tecnologia (LTSC, 2000). Segundo a IEEE/LTSC o padrão LOM (Learning Object Metadado) focaliza o mínimo de conjunto de atributos necessários para permitir que um Objeto de Aprendizagem seja gerenciado, localizado e avaliado. Os metadados permitem a catalogação e a codificação do objeto de Aprendizagem, tornando-o compreensível para as diversas plataformas.

A principal característica do objeto de aprendizagem é sua reusabilidade, que é posta em prática através de repositórios, que armazenam os objetos, permitindo serem localizados a partir da busca por temas, por nível de dificuldade, por autor ou por relação com outros objetos. Para que um objeto de aprendizagem possa ser recuperado e reutilizado, é preciso que esse objeto seja devidamente indexado e armazenado em um repositório.

\section{A Teoria Instrutiva}

Wiley (2000) afirma que os OA podem transformar a tecnologia instrutiva em linha de aprendizagem. Mas, somente os padrões técnicos não são suficientes para promover a aprendizagem. Para que isso aconteça, o uso da tecnologia deve ser guiado 
por princípios instrutivos, os quais descrevem as estratégias, as diretrizes e os critérios para a sua aplicação.

As teorias instrutivas conhecidas atualmente não dão suporte ao design e ao uso instrutivo do OA. Segundo Reigeluth e Frick (1999) apud Wiley (2000) são necessárias mais teorias que forneçam a orientação para o uso das novas ferramentas da tecnologia de informação.

Wiley (2000) apresentou uma nova teoria instrutiva criada especificamente para dar suporte ao design e a seqüência do AO, chamada Learning Object Design and Sequencing Theory (LODAS).

\section{A Teoria Instrutiva LODAS}

A teoria instrutiva "Learning Object Design and Sequencing Theory" (LODAS) de Wiley foi adaptada da metodologia de Nelson (1998) apud Wiley (2000), e é uma combinação de métodos sistematizados de diversas teorias existentes e de novas teorias. LODAS é compreendido pelos objetivos, valores, condições e métodos.

\subsection{Objetivos de LODAS}

Os objetivos foram obtidos examinando a finalidade do OA e são:

a)Verificar o uso do OA em relação ao contexto do projeto instrutivo.

b)Fornecer a sustentação explicita para o design e para a seqüência do OA.

c)Fornecer a sustentação do reuso do OA.

d)Fornecer a compatibilidade do domínio de conteúdo baseado na revisão da literatura.

\subsection{Valores de LODAS}

Os valores estão relacionados com as características desejadas na teoria e são:

a)Significância: significa a instrução possível ou os graus de precisão (ou eficiência) que não eram previamente possíveis.

b)Comunicabilidade: a teoria deve ser formalizada e explanada adequadamente que facilite sua comunicação.

c)Parsimonia: a teoria não deve ser complicada ou consumir muito tempo que impossibilite sua execução. 
d)Realizabilidade: a teoria deve ser implementada dentro da estrutura tecnológica de OA.

e)Sustentabilidade: a teoria deve inicialmente ter a tecnologia dos OA e ser compatível, a longo prazo, com uma agenda de melhoria.

\subsection{Condições para a aplicação da TEORIA}

Deve-se, inicialmente, determinar uma motivação para as necessidades do aprendiz, o estilo do instrutor, o ambiente de aprendizagem e os objetivos instrutivos.

LODAS funciona melhor em um ambiente de aprendizagem onde o instrutor e o aprendiz têm uma responsabilidade comum para a aprendizagem, e a avaliação é uma ferramenta para facilitar o progresso. Participando das avaliações formativas, os aprendizes podem monitorar e regular a sua aprendizagem. $\mathrm{O}$ instrutor deve envolver o estudante na sua própria aprendizagem e deve usar a avaliação como uma ferramenta diagnóstica. $\mathrm{O}$ instrutor e os aprendizes devem ter competência no uso do computador a fim de usar o ambiente de aprendizagem.

Para finalidades da execução, LODAS deve ser dividido em 2 seções: prescrições do projeto da instrução e prescrições do projeto OA.

\subsection{Métodos de LODAS}

Os métodos caem em seis categorias: Atividades preliminares; Análise e síntese do conteúdo; Apresentação da prática e da informação do design; Seleção e/ou projeto do O A; Seqüência do O A; Melhoria da qualidade do OA.

\subsubsection{Atividades preliminares}

O instrutor deve comparar os objetivos, os valores e as condições acima com seus próprios valores e os do ambiente. Cabe ao instrutor a decisão de aceitá-los, ou então, procurar outros que se ajuste a eles.

\subsubsection{Análise e síntese do conteúdo}

Normalmente, os conteúdos são quebrados em pequenos módulos. Sendo que esses módulos podem ser reutilizados individualmente ou combinados com outros módulos.

Os métodos que transformam o domínio do conteúdo em especificações para o espaço e a organização do conteúdo no AO são: Princípio da decomposição de 
habilidades cognitivas; Síntese do trabalho modelado (recombinação de módulos); dimensão do domínio; Escalas para o trabalho modelado (do simples para o complexo).

\subsubsection{Apresentação da prática e da informação do design}

Os componentes da habilidade cognitiva complexa podem ser classificados em um de dois grupos:

a)Habilidades recorrentes: são aquelas que são executadas na mesma maneira de cada vez (métodos algoritmos).

b)Habilidades não-recorrentes: são aquelas que diferem de acordo com a situação em que são executadas (métodos heurísticos).

Cada habilidade requer um formulário de informação prévia. Um pré-requisito necessário, como procedimentos, regras ou conceitos, para habilidades recorrentes. Ou, o conhecimento de suporte, tal como modelos casuais, conceituais ou mentais, para habilidades não-recorrentes.

A ênfase instrutiva do projeto está em encontrar problemas instrutivos e exemplos trabalhados para o modelo de interesse. Isto é realizado através da criação dos tipos de caso. Também são projetados os problemas específicos reais dentro de cada tipo de caso.

Van Merriënboer (1997) apud Wiley (2000) recomenda que os tipos de caso dentro da prática do trabalho de interesse estejam arranjados em seqüências do simples para os casos mais complexos. E para modelos de trabalhos menores a seqüência seja instrucional.

A informação é apresentada antes (dedutivo) ou depois (indutivo) de casos estudados e exemplos, mas antes da prática. A apresentação deve forçar relacionamentos significativos e a ligação do conhecimento.

\subsubsection{Selecionar e/ou projetar OA}

Wiley (2000) mostrou uma classificação dos tipos de OA disponíveis. Essa taxionomia não é exaustiva, pois incluem somente OA que facilitam o reuso. Os diferentes tipos de OA são: tipo único; combinado- inédito; combinado-modificável; gerador-apresentação e gerador-instrutivo.

Antes de iniciar o projeto instrutivo do OA, deve-se decidir qual o tipo de OA será usado. Van Merriënboer (1997) apud Wiley (2000) recomenda para as habilidades cognitivas recorrentes os $\mathrm{OA}$ do tipo gerador, mas quando as habilidades são nãorecorrentes os OA podem ser do tipo combinado. Regra geral, usar OA gerador sempre que possível, se não, usar OA combinados.

\subsection{5-Sequência do OA}


A etapa final do projeto é organizar a seqüência do OA. Essa organização em seqüência deve ocorrer em três níveis: do modelo do trabalho de interesse; dos tipos de caso e problemas específicos.

Os modelos individuais do trabalho são desenvolvidos e colocados em ordem crescente de dificuldade. $\mathrm{O}$ aprendiz deve terminar cada modelo de trabalho em um nível dado, antes de tentar modelo de trabalho no nível seguinte.

Os tipos de caso e os problemas específicos devem ser organizados em seqüência do simples para o complexo.

\subsection{6-Melhoria de qualidade do OA}

Após desenvolver a teoria instrutiva LODAS, sua implementação será realizada em três etapas: execução, avaliação e revisão.

A medida para avaliar a melhoria de qualidade é especificada no começo do projeto, e, estas guiam o projeto e a avaliação. As avaliações formativas podem ser conduzidas individuais ou usando grupos pequenos durante o processo de desenvolvimento. $\mathrm{O}$ instrutor pode observar a função de sua instrução e anotar as falhas. O projeto pode mudar ou são introduzidas intervenções de modo que o estudante possa beneficiar-se delas. Isto pode incluir o re-projeto da instrução.

\section{Proposta de aplicação da Teoria Instrutiva LODAS}

Este projeto tem por objetivo usar os princípios da metodologia instrutiva LODAS de Wiley no desenvolvimento de um Objeto de Aprendizagem para ensinar a Fórmula de Bhaskara. O Objeto de Aprendizagem proposto visa aproximar a tecnologia ao ensino, favorecendo, ao estudante, a aquisição de conhecimentos, competências e habilidades na resolução da equação do segundo grau, usando a Fórmula de Bhaskara, tornando a aprendizagem mais dinâmica e atraente. Este projeto seguirá os seguintes passos:

-Definir a finalidade do aprendizado da Fórmula de Bhaskara. Determinando também uma motivação para o aprendizado, o estilo do instrutor e o ambiente de aprendizagem.

-Definir os pré-requisitos para o aprendizado da Fórmula de Bhaskara.

-Determinar os objetivos, o domínio e as condições para o aprendizado da Fórmula de Bhaskara. Explicando o uso do objeto de aprendizagem no contexto desta teoria; fornecendo a sustentação explícita para o design e para a organização de sua sequiência; fornecendo a sustentação do reuso; fornecendo o conteúdo de Fórmula de Bhaskara, baseado na revisão de literatura.

-Desenvolver um método instrutivo apropriado e as estratégias necessárias para o aprendizado da Fórmula de Bhaskara. Esse método será desenvolvido baseado em 
modelos existentes na literatura e que prevê a identificação de um processo ótimo. Onde nenhum método for selecionado para o processo ótimo, será criado um método novo.

-Identificar as condições necessárias para fornecer as diretrizes da teoria de aprendizagem da Fórmula de Bhaskara.

-Desenvolver critérios (significância; comunicabilidade; parsimônia; realizabilidade; sustentabilidade) para avaliar a atribuição dos objetivos, dos métodos e das condições identificadas para esta teoria específica.

-Definir o tipo de OA (tipo único; combinado- inédito; combinado-modificável; gerador-apresentação e gerador-instrutivo) que será usado neste projeto.

-Criar uma taxionomia para a teoria de aprendizagem da Fórmula de Bhaskara. Serão agrupados os objetivos, os métodos e as condições em uma representação que destaca comunidades e relacionamentos.

-Criar o protótipo do OA. Esse protótipo esboça a organização e a execução do método e das estratégias. O Objeto de aprendizagem será implementado em Java Script e apresentará recursos de textos e figuras.

-Formalizar a pesquisa da teoria do protótipo. Examinar a compreensão, a coesão e a congruência da nova teoria.

-Revisar os objetivos, os métodos, e as condições. É apropriado a revisão da teoria por um expert, fornecendo um feedback detalhado da nova teoria.

-Desenvolver um plano para testar o protótipo. Este plano deve examinar a validade da teoria instrutiva.

-Escrever sobre essa teoria. Formalizar e disseminar a versão da teoria instrutiva de maneira que possa ser examinada e criticada por outros educadores.

-Executar e melhorar essa teoria.

\section{6-CONCLUSÃO}

Neste trabalho, mostrou-se que objetos de aprendizagem são materiais educacionais, suportado por computador, construidos com objetivos pedagógicos que servem para apoiar o processo de aprendizagem presencial e/ou à distância e que possuem como característica a reusabilidade, a acessibilidade, a durabilidade e a modularidade.

Os Objetos de aprendizagem podem transformar a tecnologia instrutiva em aprendizagem. Para que isso aconteça, o uso da tecnologia deve ser guiado por teorias instrutivas, as quais descrevem as estratégias, as diretrizes e os critérios para a sua aplicação. 
Espera-se que a teoria instrutiva para ensinar a Fórmula de Bhaskara, proposta neste projeto, venha proporcionar melhorias significativas no processo ensino e aprendizagem, dando condições ao estudante para participar na construção de seu próprio conhecimento.

\section{7-REFERÊNCIAS BIBLIOGRÁFICAS}

IEEE Learning Technology Standards Committee (LTSC) (2000) "Draft Standard for Learning Object Metadata", Institute of Electrical and Electronics Engineers, Inc.

LTSC. (2000). Learning technology standards committee website. Disponível em: http://ltsc.ieee.org/ Acesso em: 20 de julho de 2007.

WILEY, D. A. Connecting learning objects to instructional design theory : A definition, a metaphor, and a taxonomy . 2000. Disponível em: http://reusability.org/read/. Acesso em: 28 de abril de 2007.

WILEY, D. A. Learning object design and sequencing theory . Doctoral dissertation, Brigham Young University. 2000. 